\title{
A influência do coletivismo na decisão de escolha por restaurantes da classe A: um estudo por meio das equações estruturais*
}

\section{La influencia del colectivismo en la decisión de elección de los restaurantes de classe A: un estudio por medio de ecuaciones estructurales}

Ari Melo Mariano ${ }^{1}$ Pedro Augusto Moreira Bittar ${ }^{2}$ João Víctor da Silva Sobrinho ${ }^{3}$ Caio Pinho Paiva ${ }^{4}$ Leônidas Melo da Silva ${ }^{5}$
* Recebido em: 15/03/2015. Aprovado em: 20/05/2015.

1 Pós Doutor em Negócios Internacionais, Pós Doutor em Metodologia Científica e Estatística Avançada. Pesquisador e Professor da Universidade de Brasília e do Centro Universitário de Brasília e Líder do grupo de pesquisa Internacionalização e aplicabilidade do conhecimento científico.

2 Bacharel em Administração de Empresas pelo Centro Universitário de Brasília e empresário do setor de alimentação.

3 Graduando em Administração de Empresas pelo Centro Universitário de Brasília, pesquisador do grupo de pesquisa Internacionalização e aplicabilidade do conhecimento científico

4 Bacharel em Administração de Empresas pelo Centro Universitário de Brasília, pesquisador do grupo de pesquisa Internacionalização e aplicabilidade do conhecimento científico.

Bacharel em Administração, especialista em finanças e estudando do MBA em Administração da Getúlio Vargas. Servidor da Universidade Estadual de Santa Cruz.

\section{Resumo}

O objetivo geral deste estudo consiste em determinar em que grau existe influência do coletivismo nos fatores que caracterizam a escolha de um restaurante de "classe A" em Brasília por parte do consumidor. Inicialmente, foi realizada pesquisa bibliográfica nas bases de dados Web of Science e Scielo, na qual foram encontrados no total 3.385 artigos com as palavras-chave coletivismo e decisão do consumidor. A segunda etapa foi realizada com pesquisa descritiva de caráter quantitativo por meio das equações estruturais, calculando o valor de predição entre coletivismo e restaurantes de luxo. O programa estatístico utilizado foi o SmartPLS, que trabalha com análise multivariada. O instrumento foi validado $(F c=0,783)$ e a análise de predição foi de $30,9 \%$. Diante da confiabilidade do estudo e do resultado do beta $(-0,626)$, pode-se perceber que a influência do coletivismo por restaurantes da "classe A" foi negativa, sugerindo que quanto maior a pressão coletiva, menor a resposta, traduzida em escolha dos restaurantes de luxo na cidade de Brasília - Distrito Federal. Isso se explica na literatura pelas características do público alvo, como exclusividade.

Palavras-chave: Coletivismo. Consumo. Cultura. Hofstede. Restaurante de luxo.

\section{Resumen}

El objetivo general de este estudio es determinar hasta qué punto existe influencia del colectivismo en los factores que caracterizan a la elección de un restaurante de "clase A" en Brasilia por el consumidor. Inicialmente, se realizó una búsqueda bibliográfica en las bases de datos Web of Science y scielo, donde se encontró un total de 3.385 artículos con palabras clave colectivismo y la decisión de los consumidores. La segunda etapa fue realizado através del enfoque cuantitativo descriptivo mediante ecuaciones estructurales, donde se hizo el cálculo del valor predictivo entre el colectivismo y restaurantes de lujo. El programa estadístico utilizado fue el SmartPLS, trabajando con el análisis multivariado. El instrumento fue validado $(\mathrm{Fc}=0,783)$ y el análisis de predicción fue del $30,9 \%$. Dada la fiabilidad del estudio y los resultados del beta $(-0,626)$, puede verse que la influencia de colectivismo de restaurantes de "Clase A" fue negativo, lo que sugiere que cuanto mayor sea la presión colectiva, menor es la respuesta, aqui representada por la elección de restaurantes exclusivos en la ciudad de Brasilia, Distrito Federal. Esto se explica en la literatura por las características del mercado consumidor, como exclusividad.

Palabras-clave: Colectivismo. Consumo. Cultura. Hofstede. Restaurante de lujo. 


\section{Introdução}

Atualmente, o mundo se torna cada vez mais competitivo e aumentam as dificuldades e desafios para sobreviver em um mercado extremamente disputado e globalizado.

Segundo Anthony McGrew (1992), a globalização se refere aos processos que atuam em escala global, que atravessa fronteiras, integrando e conectando sociedades em novas combinações de espaço-tempo, fazendo com que o mundo fique mais conectado. Ela influencia alguns fatores, como a estratégia, preço e produto. Assim, a globalização afeta os países por meio das diferenças culturais, por mais tradicionais que sejam.

Segundo Castells (2007) a globalização fortaleceu os vínculos entre as fronteiras, aproximou grupos humanos de diversas etnias e promoveu transformações sociais e culturais. Desse modo, um novo cenário se desponta e passa a existir convergência de valores entre as comunidades que acabam por afetar o comportamento desses povos. Assim, realizar operações nos mercados atuais consiste em entender como a cultura afeta a tomada de decisão dos consumidores

Segundo Shimizu (2006), tomada de decisão pode ser considerada processo cognitivo, na qual a pessoa escolhe o caminho mais adequado para aquela situação ou circunstância. Esse processo decisório gera consequência, desde uma simples decisão até uma conclusão de maior impacto. O processo de decisão sofre distorções de acordo com as características pessoais de quem toma a decisão, da sua situação atual, da sua cultura e da forma como enxerga o mundo.

Hofstede (1982) define cultura como a constituição de ideias tradicionais e de valores que estão ligados ao modo de pensar, de sentir e de reagir de determinada sociedade. Muitos são os autores como Franz Boas (1858), Margaret Mead (1901) e Geertz (1977) que estudaram as mudanças e os impactos da cultura na sociedade. Entre eles se encontra Hofstede (1980), que divide a cultura em dimensões para melhor compreensão.

As dimensões de Hofstede (1980) são fundamentais para reconhecer a importância da diversificação cultural e de seus impactos em organizações. Entre as dimensões de Hofstede (1980) se encontra o Individualismo versus Coletivismo é de fundamental importância, pois, ao estar diretamente associado com valores coletivos ou individuais, altera, de maneira significativa, a per- cepção de mercado e das outras dimensões citadas por Hofstede (1980). Num amplo sentido, os homens passam a reconhecer que fazem parte de uma mesma coletividade e, assim, dentro desse contexto, as diferenças são minimizadas. Pode-se dizer que os membros dessa "coletividade" sofrem influências entre si ao ponto de provocar profundas mudanças nos seus comportamentos e tradições, ou seja, afeta a sua própria cultura.

Esse tema justifica-se para a administração, pois entender as relações entre a cultura e a decisão de consumo consiste em diminuir a possibilidade de erros em abordagem a mercados diferentes a cultura da empresa e seus executivos. Socialmente é importante, pois melhora a comunicação entre empresa e mercado local, evitando conflitos e academicamente pode-se perceber um aumento do tema ano a ano, conforme gráfico 1 .

Gráfico 1- Número de estudos ano a ano

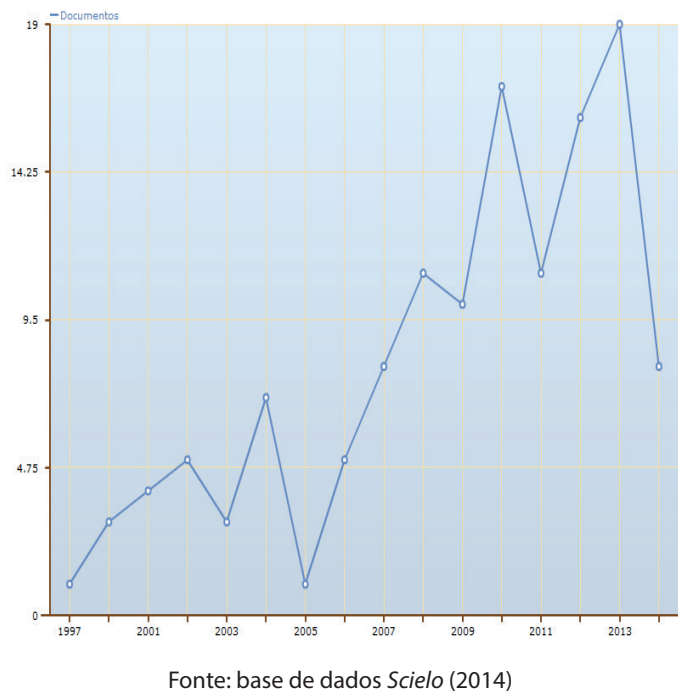

$\mathrm{O}$ aumento do número de publicações sugere aumento do interesse científico a respeito deste. A queda apresentada no ano 2014 se deve à atualização da própria base de dados que se alimenta de vários periódicos que possuem datas de publicação posteriores ao dia da coleta do gráfico (20 de novembro de 2014).

Ao observar esse contexto, surge o problema: quais as implicações da interferência do coletivismo no poder de decisão do consumidor brasileiro por restaurantes da classe A em Brasília?

Assim, o objetivo geral desse estudo consiste em determinar em que grau existe influência do coletivismo nos fatores que caracterizam a escolha de um restaurante de classe A em Brasília por parte do consumidor; e, de modo específico, objetiva apresentar indicadores associados ao coletivismo, identificar os fatores intrínsecos e ex- 
trínsecos que exercem influência no poder de escolha do consumidor no momento de escolha de um restaurante tipo "A" e determinar o grau de influência do coletivismo nos fatores que decidem a escolha de restaurantes do tipo classe A.

Para alcançar esse objetivo, foi realizado inicialmente um estudo teórico, por meio de pesquisa bibliográfica para revelar os indicadores de coletivismo e de decisão de restaurantes de classe $\mathrm{A}$, em uma segunda etapa foi realizado um estudo quantitativo de caráter descritivo por meio de questionário para apresentar o grau de influencia entre coletivismo e poder de decisão em restaurantes de classe $\mathrm{A}$.

\section{Desenvolvimento}

\subsection{Cultura}

Muitas são as abordagens que têm tentado explicar as concepções, signos e significados sobre cultura, suas interações e alcance. Na concepção de Tylor (1832, apud BARROS, 2001, p. 2), cultura é:

[...] a expressão da totalidade da vida social do homem, caracterizada pela sua dimensão coletiva, adquirida em grande parte inconscientemente e independente da hereditariedade biológica.

Em contrapartida, Franz Boas (1858), precursor da antropologia cultural norte-americana e defensora do relativismo cultural, rejeita as teorias evolucionista e difusionista de Tylor (1832, apud BARROS, 2001), uma vez que cada cultura exibe suas particularidades. Para ele, cultura não é proveniente de estágios ou fases culturais, mas se manifesta por meio da autonomia e singularidade dos povos.

A despeito das diversas abordagens em torno do assunto, sabe-se que, de acordo com a origem e consequência de sua utilização, a cultura irá variar conforme a conduta, as tradições e os costumes de cada lugar. Com base nesse conhecimento, este estudo adota a ênfase ao significado do termo, fundamentado nos estudos do psicólogo neolandês Geert Hofstede. A teoria de Hofstede (1980) é fundamentada na análise dessa dinâmica cultural. Seu estudo ressalta a importância de se observar o modo de pensar e agir de determinados grupos humanos, sobretudo em relação à distinção de comportamentos entre si, que afetam sociedades e organizações. Os estudos de Hofstede resultaram nas 5 dimensões por ele elaboradas, conhecidas como: distância hierárquica, que trata da forma como uma sociedade ou organização lida com as diferenças sociais e suas respectivas desigualdades; redução da incerteza, que se refere, basicamente, ao grau de tolerância que uma cultura pode aceitar em face de eventos inesperados; individualismo em oposição ao coletivismo. É de fundamental importância, pois tanto o individualismo quanto o coletivismo expressam as variantes do grau de liberdade de um indivíduo em relação a um determinado grupo; Masculinidade e feminilidade, que diz respeito a valores atribuídos historicamente a homens ou mulheres e maneira que influi na sociedade e orientação a longo prazo versus a orientação a curto prazo.

Deve-se considerar cada dimensão para compreender a dinâmica cultural e a interação dos valores organizacionais. A distância hierárquica diz respeito à extensão na qual certo grupo aceita o fato e convive com as desigualdades em sua organização, na qual pode-se notar maiores desigualdades, privilégios e autoridade na própria sociedade. A redução à incerteza cita a extensão na qual determinado grupo se sente ameaçado por situações ambíguas ou incertas, ou seja, é o grau de tolerância que uma cultura pode aceitar em relação a eventos inesperados e essas situações apresentam diferentes coeficientes de aversão ao risco. A dimensão individualismo versus coletivismo consiste na maneira como o ser está ligado à estrutura social integral, expressando o grau de liberdade da pessoa em relação a uma situação, esse laço pode ser mais rígida ou mais flexível, no sentido de abrir lacunas para a exibição de atitudes e de comportamentos individualistas, ou seja, a ideia do nós em contraposição ao indivíduo. Masculinidade versus feminilidade, como já foi mencionado, diz respeito a valores mais voltados ao masculino (heroísmo, determinação pessoal, sucesso material e realização) em relação a valores femininos (qualidade de vida, ênfase nos relacionamentos, cuidado com os outros e modéstia). Por fim, a orientação a longo prazo versus a orientação a curto prazo, no qual foi identificada em um trabalho posterior de Hofstede (1991), que está diretamente relacionada à expectativa de tempo de retorno em termos de resultados e recompensas de alguma ação ou tarefa realizada.

Entre essas dimensões, estudar o coletivismo como meio de influência no poder de decisão do consumidor é indispensável para determinar a estratégia de gestão a ser tomada. 


\subsection{Coletivismo versus Individualismo}

A tentativa de relacionar a pessoa com um grupo ou sociedade vem sendo pesquisada e fundamentada em diversas ciências. Durkheim (1982) falava no nível de solidariedade em diferentes aspectos, como o social. Schwartz (1990) e Triandis (1995) afirmam "o individualismo e o coletivismo podem coexistir em uma mesma organização ou, até mesmo, em apenas um indivíduo”.

Na opinião de Hofstede (1984), “o coletivismo compreende uma preferência por contextos sociais pouco fechados, onde se supõe que são os indivíduos que tem que cuidar de si mesmos, frente a uma dependência aos grupos dos quais os indivíduos fazem parte”. Ou seja, Hofstede quis identificar uma estrutura unidimensional que expressaria a independência emocional dos grupos, organizações ou qualquer outro tipo de coletividade.

Os estudos de Hofstede (1984) demonstram que existem grupos culturais que se diferenciam uns dos outros, tanto nas tradições e costumes quanto em seu aspecto comportamental. Da análise das sociedades e organizações situadas em diferentes partes do mundo e de suas dinâmicas culturais distintas, produziram-se os conceitos que são descritos neste estudo e que se aplicam ao tema estudado. Segundo o teórico, a cultura é coletiva e intangível. Ela diferencia grupos, organizações e países.

A análise desses grupos humanos e de seus comportamentos foi fundamental para chegar aos resultados acerca do estudo de Hofstede (1984). Hofstede (1984) reconhece que a importância da diversificação cultural é imprescindível para determinar não só o modo de gerir das empresas, mas também para diferenciá-las quanto ao lugar onde se situam, já que o impacto causado nessas organizações não é o mesmo quando estas se encontram em diferentes ambientes. Segundo o resultado de sua pesquisa, Hofstede (1984) concluiu que a valoração da questão humana contribui para a melhoria da gestão de empresas. Por isso, o estudo de Hofstede (1984) é de importante compreensão no âmbito em que compreende a administração, principalmente relacionar estratégias de marketing para atingir de melhor maneira o público-alvo, relacionando com os aspectos culturais que diferenciam cada sociedade.

As diferentes decisões são moldadas a partir de condutas coletivas ou exclusivas (individualismo). Schwartz (1991) relaciona qualidades ou características com tendências mais coletivas ou individualistas, ou seja, realizações financeiras, liderança e autodireção se relacio- nam com interesses individualistas. Características como a segurança, conformidade e tradição se relacionam com características coletivistas. Apesar de diferentes tendências, a pessoa em geral é moldado tanto para condutas coletivas como para exclusivas, o que diferencia uns dos outros são suas necessidades e seu processo decisório.

\subsection{Indicadores de Coletivismo}

Aaker e Williams (1998) mensuraram o efeito de apelos emocionais entre culturas e notaram a razão pelo qual esses apelos variam na sua efetividade, apelos baseados em emoções focadas nos outros, como a empatia e a paz estão relacionadas a atitudes mais coletivas, em contradição a apelos focados no ego, como o orgulho e felicidade, conduzindo assim, atitudes mais individualistas e exclusivas. Então, para medir, de maneira mais exata, algumas decisões com base nessas características, alguns indicadores como condutas coletivas e condutas exclusivas serão analisados frente aos indicadores que mensuram restaurantes da classe $\mathrm{A}$.

O primeiro indicador de coletivismo diz respeito a possuir condutas coletivas e como essas atitudes influem no subconsciente das pessoas, ou seja, serão mensuradas as ações que, muitas vezes não possuem relação com o ser. Por exemplo, alguns sentimentos que rodeiam pessoas próximas ou forte identificação com personalidades e atitudes de indivíduos ao seu redor. A lealdade ao grupo constitui ponto que tem forte relação com essas condutas coletivas. Esse fator possui forte ligação para mensurar em até que ponto o ser estaria disposto a chegar para satisfazer as necessidades do grupo em que ele está inserido. Como exemplo, podem-se citar algumas características de devoção, de esforço e de respeito com os sentimentos e atitudes alheias.

Por outro lado, a exclusividade constitui fator determinante para indicar o poder do coletivo na escolha de restaurantes de luxo, pois o sentimento de exclusividade e confortabilidade refletem valoração da pessoa frente à massificação do atendimento, dos serviços e dos produtos oferecidos. Para Velho (1987) e Dumont (1985), as relações humanas trazem traços mais individualistas, nesse contexto, ações individuais são voltadas para a promoção de orientações sociais e disposições psicológicas, nas quais as pessoas se orientam exclusivamente para o próprio bem-estar em relação aos outros e de suas condutas mais coletivas.

Então, com base nos indicadores, pode-se relacio- 
nar a lealdade e a exclusividade com o coletivismo, mensurando, assim, pontos mais voltados a condutas coletivas ou a condutas individualistas.

Esses indicadores da dimensão coletivismo exercem influência sobre o poder de decisão do consumidor.

\subsection{Poder de decisão e as reais necessidades do con- sumidor}

Kotler e Armstrong (1993) destacam três fatores que podem influenciar a tomada de decisão e comportamentos do consumidor:

1. motivações: as necessidades são divididas em fisiológicas (fome, sede, desconforto) e psicológicas (reconhecimento, autoestima e relacionamento). Os consumidores são influenciados por essas necessidades, mas elas não são suficientes para motivar o indivíduo a agir em busca de sua satisfação, é necessário um motivo maior.

2. personalidade: o comportamento do consumidor é determinado pelas diferentes personalidades de cada ser, pois refere-se às características psicológicas que levam a uma resposta de acordo com o ambiente no qual o indivíduo está inserido. O comportamento do consumidor frente a uma marca ou produto é analisado para decifrar sua personalidade.

3. percepções: considerada como "processo pelo qual as pessoas selecionam, organizam e interpretam informações para formar uma imagem significativa do mundo" (KOTLER; ARMSTRONG, 1993, p. 89).

Diante desses três fatores, pode-se reconhecer quais as reais necessidades e quais os diferentes posicionamentos que o consumidor possui frente a uma marca ou produto, afetando assim a sua decisão final. Chiavenato (2011) descreve que o consumidor busca alternativas para poder escolher e diagnosticar o problema da melhor maneira possível, identificando suas reais soluções, assim escolhendo a alternativa mais adequada para o alcance dos objetivos traçados.

Assim, cada pessoa toma decisões baseadas em aspectos subjetivos e essa subjetividade não possui medida perfeita, criando assim diferentes decisões. As escolhas não podem ser taxadas como certas ou erradas, mas suas consequências podem e devem ser analisadas e revistas em situações semelhantes.

A partir do pressuposto dessas relações, Schwartz (1992) acredita que os valores humanos assumem orientações segundo os interesses que cumprem, ou seja, de acordo com um enquadramento de certa sociedade, o grupo correlaciona-se com os valores pessoais (indivi- dualismo) ou com os valores sociais (coletivismo) e esses valores humanos ajudam a definir a tendência a ser seguida da pessoa e, consequentemente, do grupo. Com isso, sociedades que priorizam os valores humanos e valores sociais, como o Brasil, tomam decisões moldadas com base em seus princípios, afetando suas decisões. A atitude está relacionada com a decisão da pessoa, e possui diversos componentes motivacionais. Por isso, o poder de decisão do ser é composto por diversas variáveis.

O comportamento do consumidor não é apenas influenciado pela cultura, mas, também, pelo processo de escolha, desde a determinação da necessidade ou desejo de um produto ou serviço, até o seu pós compra, se atendeu às necessidades e expectativas exigidas. Segundo Kotler (1998), a área do comportamento do consumidor estuda como pessoas, grupos e organizações selecionam, compram, usam e descartam produtos, serviços, ideias ou experiências para satisfazer suas necessidades e seus desejos.

Embora sejam evidentes as diferenças de sentimentos e necessidades de cada ser, o processo decisório que faz com que a pessoa escolha determinado produto é praticamente o mesmo, o que deve diferenciar consiste na percepção de cada observador frente à marca, produto ou serviço. Entretanto, deve-se identificar se essa percepção e positiva ou negativa. Por essa razão, há necessidade de indagar diretamente ao consumidor acerca de algo que lhe está sendo ofertado. Portanto, por meio do reconhecimento da visão de mundo dos indivíduos e do estudo das forças culturais e sociais que lhe dão forma, pode-se compreender melhor como os consumidores criam novos sentidos para objetos, produtos ou serviços que lhes são ofertados.

\subsection{Restaurantes da Classe A: definição e caracte- rísticas}

A classe social pertencente de grupo é definida com base em status social similar segundo vários fatores, em especial o econômico. Para Marx (1867), quando este menciona-se classe social e suas desigualdades, refere-se ao poder aquisitivo, ao acesso à renda, à posição social, ao padrão de vida, entre outros.

Segundo o DIEESE (Departamento Intersindical de Estatística e Estudos Socioeconômicos), foi realizado estudo para definir a classificação do ser perante a sociedade. A classificação afirma que pessoas pertencentes à classe $\mathrm{A}$ recebem vinte ou mais salários mínimos. Já a 
FGV (Fundação Getúlio Vargas) as famílias são definidas por uma faixa estabelecida que determina a renda familiar destas, com isso, famílias que recebem acima de $\mathrm{R} \$ 9.745,00$ são pertencentes à classe social mais elevada da sociedade brasileira (apenas 3\% da população brasileira).

Com base nesses dados, pode-se notar que a classe A é absoluta minoria no Brasil, mas é nela que estão os indivíduos mais exigentes em relação a algum serviço ou produto, pois estão acostumados com algumas regalias e um padrão de vida mais sofisticado. E no ramo da alimentação não é diferente, os consumidores de estabelecimentos da classe A exigem alguns aspectos que superam suas características, por isso a definição de restaurante classe A também é dada a partir de uma série de fatores que faz com que o consumidor esteja plenamente satisfeito com o estabelecimento em geral.

Brasília é uma cidade com poder aquisitivo alto, conforme dados do Instituto Brasileiro de Geografia e Estatística (IBGE), Brasília tem uma das maiores rendas per capita do Brasil com cerca de R $\$ 1.715,11$. Com isso, o número de restaurantes desenhados para atender um público mais exigente e diversificado é alto. Como a capital do país possui, segundo o Ministério das Relações Exteriores, 184 embaixadas e diversos visitantes de todas as partes do país e do mundo, é de fundamental relevância estudar fatores culturais associados ao poder de decisão desses clientes.

Esse grupo de clientes considerados "Classe A" possuem características especiais e segundo Faria (2007), eles que delimitam o que o estabelecimento deve ter para ser considerado classe $\mathrm{A}$, ou seja, o enquadramento dos restaurantes deve ser norteados pelas exigências dos clientes, pois são eles que podem, pedem e pagam por serviços de excelência. Existem três enfoques que dão suporte aos clientes que consomem na classe A:

\section{1.ética}

2. comprometimento

3. orgulho

O cliente exige tratamento diferenciado, com a sofisticação do seu próprio padrão de vida. Assim, a ética deve estar presente na relação estabelecimento-consumidor, pois deve-se ter transparência total e integral com cada cliente. As exigências desse público são maiores, mas deve-se prometer apenas aquilo que o restaurante pode cumprir (comprometimento). Com o prevalecimento da ética e do comprometimento do restaurante com o cliente, naturalmente o orgulho surge. $\mathrm{O}$ estabelecimento é reconhecido pela excelência dos serviços prestados e, em consonância, o cliente admite que tal estabelecimento corresponde às mais exigentes expectativas.

Portanto, algumas qualidades e características peculiares de cada restaurante da classe A como produto, serviço, localização e público frequentador, não são suficientes, pois cada consumidor possui necessidades diferentes, muitas vezes bem parecidas. Porém, aliando à ética e ao comprometimento em todos os processos, o estabelecimento irá corresponder e superar todas as expectativas, pois aliar qualidade e confiança em repassar essa qualidade é fundamental.

\subsection{Indicadores de restaurantes de luxo}

Segundo Hennigs (et al. 2012), restaurante de luxo é definido indicadores para mensurar algumas dimensões acerca do consumo em geral dos bens de luxo. Grandes empresas de luxo têm como desafio identificar e satisfazer as necessidades dos mais exigentes consumidores e isso é definido como grande desafio, pois o modelo de consumo tradicional foi transformado em nova sensibilidade, mudando, também a concepção da palavra "luxo". Várias pessoas de diferentes culturas e por motivos variados frequentam, consomem ou compram produtos de luxo, mas essas pessoas possuem valores semelhantes e partem de quatro fatores motivacionais básicos que foram mapeados no presente estudo.

O primeiro ponto constitui o valor financeiro, pois é necessário definir e analisar uma variável que molda se esse aspecto é relevante para frequentar restaurantes de luxo e se o valor financeiro é realmente um fator que delimita o público que frequenta o estabelecimento. Cabe analisar, dentro dessa dimensão, se o bairro em que o restaurante está inserido é determinante para a escolha do restaurante de luxo.

Outras duas dimensões fundamentais para determinar a escolha do restaurante de luxo são o valor individual e o valor social. Seres com características individuais possuem fatores prioritários a serem atendidos, como a autossatisfação e o prazer próprio. Em consequência disso, as soluções mais plausíveis são voltadas exclusivamente para seus problemas. Já no valor social, o status ao qual a pessoa pertence e as boas impressões sobre os outros constituem prioridade. Nesse valor, as percepções dos outros se tornam mais relevantes e suas influências afetam o processo decisório. A autossatisfação e identidade pessoal são aspec- 
tos mensurados em estudos semelhantes que visam medir a interferência do coletivo nas decisões de consumo de frequentadores de restaurantes de luxo. Será dado enfoque aos processos que cercam a pessoa acerca de influenciar ou não em suas decisões. Não se deve deixar de considerar que, muitas vezes, a satisfação e a boa impressão que determinados restaurantes causam nas pessoas são repassadas, assim, a tendência é um indivíduo com percepções e gostos parecidos ter opinião semelhante. Com isso, alguns pontos deixam de ser tão tendenciosos, pois as interpretações e as prioridades de uma pessoa com valores e personalidades semelhantes se tornam, praticamente, iguais.

Por último, o valor funcional consiste outro ponto que deve ser analisado no que diz respeito à escolha de determinado restaurante de luxo. É com ele que se pode medir as principais qualidades do estabelecimento e essas qualidades são provindas de atendimento ou produtos diferenciados. O valor funcional, se destacado, demonstra que a pessoa está satisfeita, não levando em consideração aspectos externos e opiniões alheias, tendo como prioridades as suas necessidades e suas exclusividades em particular.

Então, os valores descritos (funcional, social, financeiro e individual) serão analisados com seus determinados pesos e aspectos aliados à teoria aplicada. De acordo com as prioridades e necessidades do ser, serão analisados os resultados, assim, qual o maior e o menor determinante para a escolha de um restaurante de luxo.

\section{Metodologia}

O método adotado neste estudo foi o descritivo, de abordagem quantitativa por meio das equações estruturais. A pesquisa descritiva, segundo Marconi e Lakatos (2010), não procura explicar o fenômeno investigado, visa descrevê-lo e contribuir para outras pesquisas que visam explicar o fenômeno investigado. $\mathrm{O}$ estudo quantitativo segundo Ganga (2012, p. 208) "é predominantemente utilizada nas ciências naturais, que se utiliza de métodos experimentais, que objetivam estabelecer relações causais entre as variáveis de pesquisa”.

Por meio da base teórica, foram identificadas dimensões que serviram para desenvolver um questionário com 33 perguntas baseadas no coletivismo e nas influencias sobre alguns aspectos que levam o cliente a decidir sobre a escolha do estabelecimento de luxo. A pesquisa foi aplicada aos frequentadores do restaurante "O Sushi- man Sushi Express", situado no Setor de Autarquias Sul quadra 3 em Brasília. A amostra utilizada foi de conveniência com 130 participantes.

A primeira dimensão analisada no estudo, o coletivismo, é formada por 13 indicadores. A segunda, restaurante de luxo, por 4 indicadores (valor financeiro, individual, social ou funcional). Todas as perguntas utilizaram a escala de Likert de 5 itens, sendo eles: Discordo, Discordo Moderadamente, Neutro (nem discordo, nem concordo), Concordo Moderadamente e Concordo. $\mathrm{O}$ aspecto de inclusão foi de frequentadores de restaurantes de luxo de uso e que tenham aceitado participar da pesquisa na data estabelecida, o índice de confiabilidade que valida o questionário foi de $(F c=0,783)$, a literatura aceita em estudos iniciais valores acima de 0,7 (RAMIREZ; MARIANO; SALAZAR, 2014).

A técnica estatística utilizada consiste em equações estruturais que trabalha com análise multivariada, utilizando o SmartPLS. Os modelos de equações estruturais utilizam modelos estatísticos de múltiplas variáveis que permite avaliar o grau de correlação que cada variável tem entre as outras (MARTÍN, et al, 2010). Os dados obtidos dos questionários foram lançados e organizados em uma planilha no Excel, como é requerido para a utilização do programa SmartPLS. Com isso o programa calculou o modelo criado, gerando informações de correlações múltiplas entre as variáveis latentes.

\section{Resultados}

Para analisar o modelo, Chin (1998) explica que, inicialmente, deve-se criar o modelo estrutural com base nas relações explicadas na literatura. Essas interpelações podem ser explicadas por meio de figura formada por nomogramas na qual as setas representam relações entre as variáveis (Figura 1).

Figura 1- Modelo Estrutural

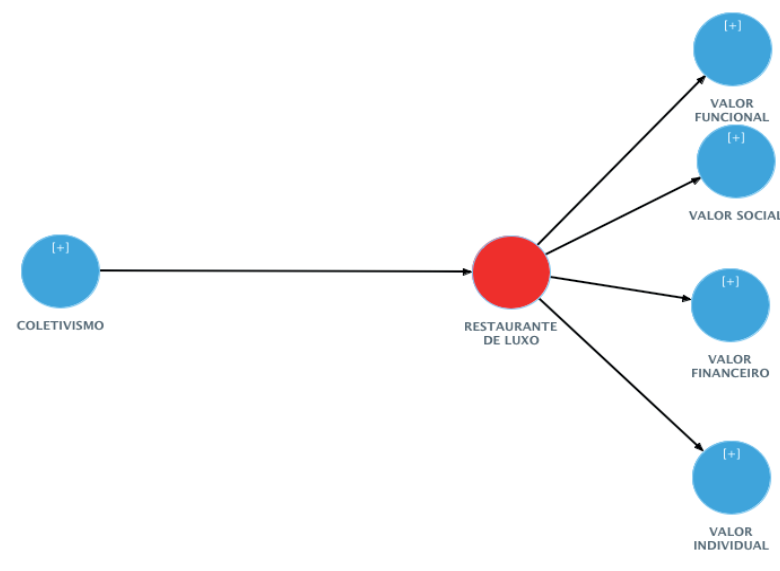

Fonte: Do autor 
O modelo apresentado pelo problema constitui a influência da variável coletivismo nos restaurantes de luxo. Porém, conforme se observa na figura acima, os restaurantes de luxo têm como antecedentes as variáveis valor funcional, financeiro, social e individual, ou seja, a variável restaurante de luxo não é medida por indicadores e sim por outras variáveis, gerando o efeito dentro das equações estruturais de constructo de segunda ordem. Ou seja, deve ser calculada uma etapa, para depois calcular o modelo total.

Pode-se observar que o nomograma em vermelho não permite que o modelo seja calculado. Para resolver esse tipo de impasse, (CHIN et al, 1996) aconselha o uso de componentes hierárquicos. Ou seja, inicialmente considera as relações da variável restaurante de luxo como as relações das suas próprias variáveis. Foi realizada a aproximação passo a passo (LOHMOLLER, 1989).

Assim o modelo foi calculado por meio de construto de segunda ordem. Pode-se observar na figura 2 que o efeito restaurante de luxo não aparece, são apresentadas suas variáveis. Uma vez calculado o modelo, utiliza-se os LatentVariablesScore para gerar os indicadores que vão formar parte do conceito de restaurantes de luxo.

O modelo é formado por figuras interligadas entre si de acordo com as relações reveladas na pesquisa bibliográficas. Os modelos são formados por círculos (nomogramas) e retângulos. Os retângulos representam as perguntas ou indicadores (itens) do questionário relacionados às suas variáveis latentes (círculos) as quais explicam. Pois parte-se do conceito que algumas variáveis não podem ser explicadas diretamente (latentes), sendo necessários indicadores para medi-las (itens). O constructo é representado pelo conjunto de itens e sua respectiva variável latente. Os números presentes nas setas que ligam as variáveis aos itens são valores de confiabilidade, expressos por correlação simples.

Figura 2 - Modelo de equações estruturais

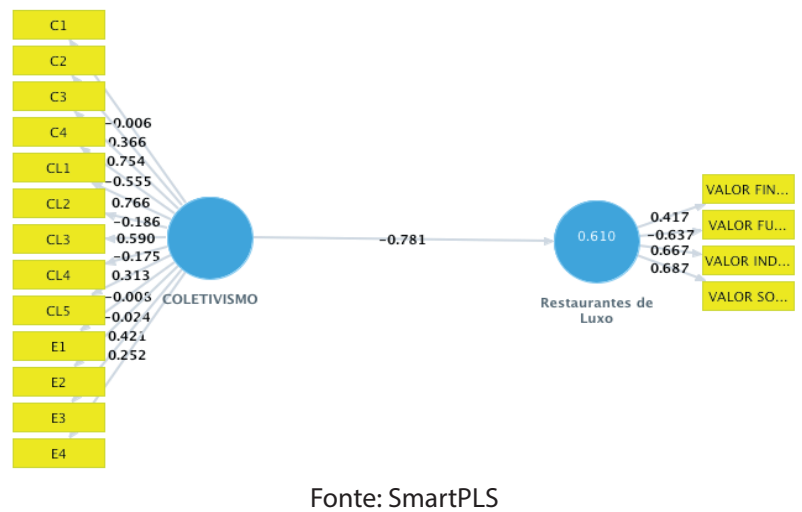

Uma vez desenhado o modelo e atribuído seus dados é necessário seguir duas etapas para sua análise. A primeira relacionada à confiabilidade do modelo e respectivamente de seu instrumento e outra relacionada ao grau de predição das variáveis dependentes e o grau de influencia das variáveis independentes.

A literatura explicita (RAMIREZ; MARIANO; SALAZAR, 2014) que para obter-se boa correlação dos itens e suas respectivas variáveis os valores devem ser maiores ou iguais a 0,6 , assim, os valores abaixo de 0,6 devem ser descartados do modelo. Essas depurações ocorrem de um a um, até o modelo se tornar estável:

Figura 3 - Modelo de equações estruturais ajustado

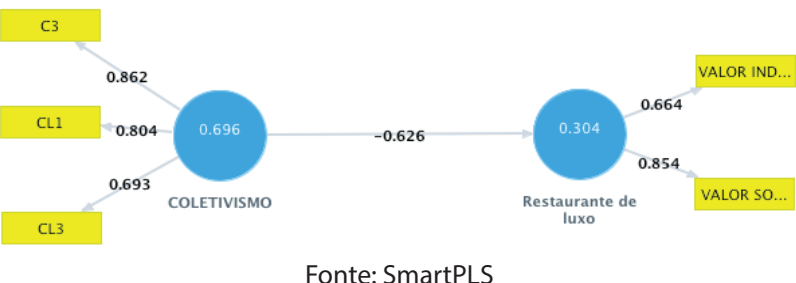

Como pode ser observado na figura 3 , os itens representados pelas setas entre os retângulos e os círculos apresentam fiabilidade maior que 0,6. Chin (1998) explica que as cargas devem ser ao menos $0.60 \mathrm{em}$ pesquisas iniciais, sugerindo que os itens presentes em forma de perguntas do questionário estão correlacionados com sua variável latente explicando-a.

O segundo teste de confiabilidade consiste no Coeficiente de Confiabilidade. Ele está relacionado como os itens agrupados a cada variável, mostrando consistência interna. Como observado na tabela abaixo, o resultado da pesquisa apresenta coeficiente de confiabilidade, superiores a 0,7 (RAMIREZ; MARIANO; SALAZAR, 2014). Esses valores mostram que o modelo é fiável e que os indicadores utilizados na pesquisa medem bem suas variáveis latentes:

Quadro 1 - Confiabilidade composta

\begin{tabular}{|c|c|}
\hline \multicolumn{2}{|c|}{ Composite reliability } \\
\hline Coletivismo & 0,831 \\
\hline Restaurante de luxo & 0,736 \\
\hline
\end{tabular}

Uma vez superada a confiabilidade de item e a composta, pode-se se dizer que o modelo é confiável. Para realizar os testes de validade é necessário calcular os coeficientes de variância média explicada e variância discriminada. 
A variância média explicada serve para analisar a validez convergente do construto (RAMÍREZ; MARIANO; SALAZAR, 2014), isso significa que os itens estão medindo a sua variável latente e não outra variável presente no modelo. Valores de AVEs devem ser acima de 0.50 (FORNELL; LARCKER, 1981; CEPEDA; ROLDÁN, 2004) para expressar validade comprovada. Os AVEs apresentados estão acima de 0.5 , ou seja, mais de $50 \%$ da variância do construto é devida aos seus indicadores:

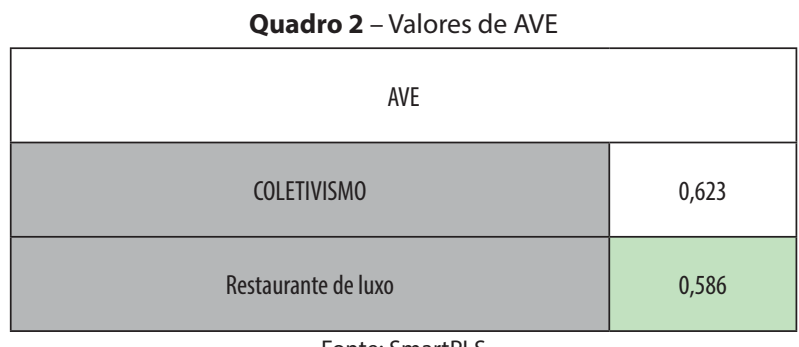

Fonte: SmartPLS

Uma vez calculada a variância explicada, mede-se a variância discriminante. $\mathrm{O}$ coeficiente de variância discriminante indica o grau que um constructo é diferente de outros (CEPEDA; ROLDÁN, 2004).

Quadro 3 - Variância discriminante

\begin{tabular}{|c|c|c|}
\hline & COLETIVISMO & Restaurante de luxo \\
\hline COLETIVISMO & 0,790 & \\
\hline Restaurante de luxo & $-0,626$ & 0,765 \\
\hline
\end{tabular}

Então, pode-se notar que o construto coletivismo é pouco relacionado com o construto restaurante de luxo. A variância discriminante é obtida por meio da Raiz quadrada de Ave (índice calculado anteriormente) comparada aos pesos das outras variáveis. $\mathrm{O}$ valor em vermelho deve ser superior aos valores abaixo.

Uma vez aprovado o modelo por sua validade e instrumento, se procede ao calculo das predições e relações.

A variável R2 indica a porcentagem em que um construto anterior explica uma variável dependente. A R2 constitui uma medida do poder de predição de um modelo (CEPEDA; ROLDÁN, 2004). A variável restaurante de luxo prediz o modelo em $30,9 \%$, considerando que o valor de R2 mínimo a ser considerado é 0.1 (FALK; MILLER, 1992). Ainda Falk e Miller (1992) colocam que valores menores que 0.1 não são considerados porque trazem pouca informação referente à variável latente. Com isso, pode-se constatar que a variável latente apresentada é bem explicada pelo constructo que as predizem (aparece na figura 3 dentro do circulo azul que recebe a seta).

Assim pode-se perceber que a decisão em frequentar um restaurante de Luxo é predita em 30,9\% pelo coletivismo.

Uma vez observado os valores de predição de R2, é o momento de apresentar a influencia de cada variável na variável predita. $\mathrm{O}$ valor beta representa o coeficiente path ou peso de regressão padrão, que são observados no modelo pelas flechas que ligam cada constructo (CEPEDA; ROLDÁN, 2004). Segundo Chin (1998, apud CEPEDA; ROLDÁN, 2004) o valor de beta deve ser no mínimo 0.2 e o ideal seria acima de 0.3 . Nota-se que o valor de beta $(-0,626)$, expresso na figura 3 anteriormente, ficou abaixo do aceito, explicando que a influência da relação coletivismo é pouco significante na predição desse constructo (restaurante de luxo) nessa situação.

Pode-se perceber que a influência é negativa. Isso se pode explicar, pois quem vai a um restaurante de luxo quer se sentir único, assim quanto maior o coletivismo, mais baixa a influência por decisões em restaurantes classe A. Faz parte da classe A descobrir e poder falar que é o único entre seus amigos que foram em um restaurante "Classe A".

\section{Considerações finais}

Este estudo analisou qual a relação existente entre a dimensão coletivismo sobre a escolha dos restaurantes de luxo. Foram apresentados breves conceitos sobre cultura e dimensões culturais a fim de mencionar e estudar a dimensão coletivismo de Hofstede. Após o embasamento teórico, foram mensurados indicadores para saber quais são as variáveis que incidem sobre restaurantes de luxo (valor financeiro, social, funcional e individual) e coletivismo (lealdade, fidelidade e exclusividade). Após a formulação dos indicadores, desenvolveu-se questionário com 33 perguntas, com a aplicação deste para clientes do restaurante "O Sushiman Sushi Express", situado no Setor de Autarquias Sul quadra 3 em Brasília.

O objetivo geral do estudo foi realizado, pois era necessária a determinação de qual o grau de influência do coletivo nos fatores que caracterizam a escolha do restaurante de classe A, foi encontrado um valor de 39,2\%. Assim como os objetivos específicos que objetivava apresentar indicadores associados ao coletivismo, os fatores intrínsecos e extrínsecos que exercem influência no po- 
der de escolha do consumidor e o grau de influência do coletivismo nos fatores que incidem a essa decisão, foi alcançado.

O modelo analisado no programa SmartPLS (SmartPartial Least Square) foi validado e fica disponível para a literatura. Foi importante entender essa predição, pois ela explica consideravelmente, porém seu grau de influência é negativo, podendo-se sugerir que valores coletivismo não estão relacionados às marcas de classe $\mathrm{A}$, porém o coletivo é bastante influenciado pelo poder dessas marcas.

Os fatores limitadores da pesquisa se deram em relação à aplicação do questionário, pelo tempo escasso e na possibilidade de oferecer amostra maior para a literatura. Para futuros estudos se aconselha a continuação e extensão desta pesquisa por meio do instrumento validado $(\mathrm{F} c=0,783)$.

\section{Referências}

AAKER, Jennifer L; WILLIAMS, Patti. Empathy versus pride: the influence of emotional appeals across cultures. Journal of Consumer Research, Chicago, v. 25, p. 241-261, dec. 1998.

BARROS, Laraia Roque. Cultura: um conceito antropológico. Rio de Janeiro: Zahar, 2001.

CASTELLS, Manuel; HUTTON, Will; GIDDENS, Anthony. Global capitalism. Economic strategy, n. 3, maio, 2000 .

CASTRO, M. Carmen Barroso; CARRIÓN, Gabriel A. Cepeda; ROLDÁN, José L. Investigar en economía de la empresa: ¿partial least squares o modelos basados en la covarianza? In: CONGRESO ANUAL, 19; CONGRESO HISPANO FRANCÉS DE AEDEM, 15, 2005, Vitoria. El comportamiento de la empresa ante entornos dinâmico: ponencias. Madrid: AEDEM, 2007. p. 63-73.

CEPEDA, Gabriel; ROLDÁN, José L. Aplicando en la práctica la técnica PLS en la Administración de Empresas. In: CONGRESO NACIONAL ACEDE, 14, 2004, Murcia. Conocimiento y Competitividad. Murcia: Universidad de Murcia, 2004. p. 74-78.

CHIAVENATO, Idalberto. Introdução à teoria geral da administração. Rio de Janeiro: Elsevier, 2011.
CHIN, Wynne W. Commentary: issues and opinion on structural equation modeling. MIS Quartely, Minnesota, v. 22, n. 1, p. 7-16, mar. 1998.

DEPARTAMENTO INTERSINDICAL DE ESTATÍSTICA E ESTUDOS SOCIOECONÔMICOS. Classificação de acordo com renda familiar. Disponível em: <http:// www.dieese.org.br/>. Acesso em: 12 set. 2014.

DUMONT, Louis. O individualismo: uma perspectiva antropológica da ideologia moderna. Rio de Janeiro: Rocco, 1985.

DURKHEIM, Émile. Da divisão do trabalho social. Madrid: Akal, 1982.

FARIA, Carlos Alberto. Serviços da classe A. 2007. Disponível em: <http://www.merkatus.com.br/10_boletim/203.htm>. Acesso em: 4 out. 2014.

FERREIRA, Aurélio Buarque de Holanda. Mini Aurélio: o minidicionário da língua portuguesa. 6. ed., rev. e atual. Curitiba: Positivo, 2004.

FORNELL, C.; LARCKER, D. F. Structural equation models with unobservable variables and measurement error: algebra and statistics. Journal of Marketing Research, Chicago, v. 18, n. 2, p. 39-50, fev. 1981.

FREITAS, Maria Ester. Cultura organizacional grandes temas em debate. Revista de Administração de Empresas, São Paulo, v. 31 n. 3, p. 73-82, maio, 1991.

FREVERT. Tonnies. The pe-redox concept in natural sediment-water systems; its role in controlling phosphorus release from lake sediments. Archiv für Hydrobiologi, Supplement, Stuttgart. n. 55, p. 278-297, 1979.

FUNDAÇÃO GETÚLIO VARGAS. Qual a faixa de renda familiar das classes?. Rio de Janeiro, 2011. Disponível em: <http://cps.fgv.br/node/3999>. Acesso em: 12 set. 2014.

GANGA, Gilberto Miller Devós. Trabalho de Conclusão de Curso na Engenharia de Produção: um guia prático de conteúdo e forma. São Paulo: Atlas, 2012.

GEERTZ, Clifford. Found in translation: on the social history of the moral imagination. The Georgia Review, Athens, v. 31, n. 4, p. 788-810, abril, 1977. 
GOUVEIA, Valdiney V. A natureza dos valores discrepantes do individualismo e coletivismo: uma comparação intercultural, 1998. f. Tese (Doutorado) - Faculdade de Psicologia, Universidade Complutense de Madri, Madri, 1998.

HENNIGS, Nadine et al. What is the value of luxury? a cross-cultural consumer perspective. Psychology and marketing, New Jersey, v. 29, n. 12, p. 1018-1034, dez. 2012.

HOFSTEDE, Geert H. Culture and organizations. Internacional Studies of Management \& Organization, London, v. 10, n. 4, p. 15-41, agosto, 1981.

HOFSTEDE, Geert H. Culture's consequences: comparing values, behaviors, institutions and organizations across nations. 2. ed. Thousand Oaks: Sage, 2001.

HOFSTEDE, Geert H. Culture's consequences: international diferences in work-related values. Newbury Park: Sage, 1984. (Cross-Cultural Research and Methodology Series, 5)

KOTLER, Philip. Administração de marketing: análise, planejamento, implementação e controle. 5. ed. São Paulo: Atlas, 1998.

KOTLER, Philip; ARMSTRONG, Gary. Princípios de marketing. 5. ed. Rio de Janeiro: Prentice Hall Brasil, 1993.

LAKATOS, Eva Maria; MARCONI, Marina Andrade. Fundamentos da metodologia científica. 3. ed. rev. e ampl. São Paulo: Atlas, 1991.

LOHMOLLER, Jan-Bernd. Latent variable path modeling with partial least squares. Heidelberg: Physica-Verlag, 1989.

MARX, Karl. Capital: a critical analysis of capitalist production. Moscow: Progress Publishers, 1867. v. 1.

MASLOW, Abraham H. Motivation and personality. New York: Harper, 1970.

MCGREW, Anthony. A global society? In: HALL, Stuart; HELD, David; MCGREW, Tony (Ed.). Modernity and its futures. Cambridge: Polity, 2003. v. 4. p. 61-116.
MEAD, Margaret. Coming of age in Samoa: a psychological study of primitive youth for western civilization. New York: American Museum of Natural History, 1973.

RAMÍREZ, Patricio E.; MARIANO, Ari Melo; SALAZAR, Evangelina A. Propuesta Metodológica para aplicar modelos de ecuaciones estructurales con PLS: el caso del uso de las bases de datos científicas en estudiantes universitarios. 2014. Disponível em: <http://www.admpg.com. br/revista2014_2/Artigos/15\%20\%20-\%20Artigo_15. pdf>. Acesso em: 08 out. 2014.

SCHWARTZ, Shalom H. Beyond individualism/collectivism: new culture dimensions of values. In: UICHOK, Kim et al. (Ed). Individualism and collectivism: theory, method and applications. Thousand Oaks: Sage, 1994. p. 85-122. (Cross-Cultural Research and Methodology Series, 18).

SCHWARTZ, Shalom H. Individualism-collectivism: critique and proposed refiments. Journal of Cross-Cultural Psychology, London, v. 21, n. 2, p. 139-157, jun. 1990.

SCHWARTZ, Steven J.; BURGESS, David. Quasi-parallel shocks: a patchwork of three-dimensional structures. Geophysical Research Letters, Washington, D.C, v. 18, n.3, p. 373-376, mar. 1993.

SHIMIZU, Nobuyuki; HAAS, Andrew R. Learning to follow navigational route instructions. In: INTERNATIONAL JOINT CONFERENCE ON ARTIFICIAL INTELLIGENCE, 21. 2009. Pasadenia. Proceedings. Pasadenia: IJCAI, 2009. v. 9, p. 1488-1493.

TONNIES, Ferdinand. Community and society $=\mathrm{Ge}-$ meinschaft und gesellschaft. New York: Dover, 2002.

TRIANDIS, H. C.; BHAWUK, D. P.; GELFAND, M. J. Horizontal and vertical dimensions of individualism and collectivism: a theoretical and measurement refinement. Cross-cultural research, London, v. 29, n. 3, p. 240-275, ago. 1995.

TRIANDIS, Harry C. et al. Converging measurement of horizontal and vertical individualism and collectivism. Journal of Personality and Social Psycology, Washington, v. 74, n. 1, p. 118-128, jan. 1998. 
TRIANDIS, Harry C. Theoretical and methodological approaches to the studt of collectivism and individualism. In: UICHOK, Kim et al. (Ed.). Individualism and collectivism: theory, method and applications. Thousand Oaks: Sage, 1994. p. 85-122. (Cross-Cultural Research and Methodology Series, 18).
UICHOK, Kim et al. (Ed.). Individualism and collectivism: theory, method and applications. Thousand Oaks: Sage, 1994. p. 85-122. (Cross-Cultural Research and Methodology Series, 18).

VELHO, Gilberto. Individualismo e cultura. Rio de Janeiro: Zahar, 1987. 